\title{
Numerical Simulation and Experimental Study for the Impact of In-Flow Nozzle on Spray Characteristics
}

Mahmoud Abd El-Aziz Mohamed ${ }^{1 *}$, Hesham El-Sayed Abdel Hameed ${ }^{1}$, ElShenawy A. ElShenawy $^{2}$, Hafez Abdel Aal El-Salmawy ${ }^{1}$, Ramy Elsayed Shaltout ${ }^{1}$

${ }^{1}$ Department of Mechanical Power Engineering, Faculty of Engineering, Zagazig University

${ }^{2}$ Department of Mechanical Power Engineering, Faculty of Engineering, Tanta University, Egypt

\section{Appendix-A}

\section{Computational Model Description}

\section{In Flow Simulation}

For the mixture flow, the phase continuity equation can be written as:

$\frac{\partial}{\partial t}\left(\alpha \rho_{q}\right)+\nabla \cdot\left(\alpha \rho_{q} \vec{u}_{q}\right)=\dot{m}_{n e t}$

Where $(q)$ is the phase type. The source term $\dot{m}_{\text {net }}$ represents the net phase change rate

$$
\dot{m}_{n s t}=\dot{m}^{+}-\dot{m}^{-}
$$

The terms $\left(\dot{m}^{+}\right)$and $\left(\dot{m}^{-}\right)$represents the effects of evaporation and condensation rates respectively as a result of the phase change, which is bubble formation due to cavitation or bubble collapse. The evaporation and condensation rates are derived from the bubble dynamics equation, which accounts for bubble growth and collapse. They can be calculated based on Rayleigh-Plesset equation as illustrated in equations (S10) and (S11). Also the sum of the phase's volume fractions satisfies the physical constraint defined by the following expression:

$\sum_{p=1}^{n} \alpha_{q}=1$

Regarding the mixture flow continuity equation, it takes the following form:

$$
\frac{\partial \rho}{\partial t}+\nabla \cdot(\rho \vec{v})=0
$$

Where the mixture quantities are defined as follows:

- Volume-Averaged mixture density 
$\rho=\sum_{q=1}^{n} \alpha_{q} \rho_{q}$

- Mass-Averaged Mixture Velocity

$\vec{v}=\frac{1}{\rho} \sum_{q=1}^{n} \alpha_{q} \rho_{q} \vec{v}_{q}$

In a flowing liquid with no slip between the liquid and the vapor phase, the generalized bubble dynamics equation can be expressed as follows ${ }^{1}$ :

$R_{B} \frac{d^{2} R_{B}}{d t^{2}}+\frac{3}{2}\left(\frac{d R_{B}}{d t}\right)^{2}=\left(\frac{P_{B}-P}{\rho_{l}}\right)-\frac{4 \mu_{l}}{\rho_{l} R_{B}} \frac{d R_{B}}{d t}-\frac{2 S}{\rho_{l} R_{B}}$

According to the above equation, the bubble radius $\left(R_{B}\right)$ depends on the density of the liquid ( $\left.\rho_{l}\right)$, the surface tension $(S)$ and the liquid viscosity $\left(\mu_{l}\right)$. The pressure $(P)$ is the cell center pressure. On the other hand, the bubble surface pressure $\left(P_{B}\right)$ equals to the vapor pressure at the corresponding temperature $\left(P_{v}\right)$.

Accordingly, both $\left(\dot{m}^{+}\right)$and $\left(\dot{m}^{-}\right)$can be calculated as follows:

$$
\begin{array}{ll}
\dot{m}^{+}=\frac{\rho_{v} \rho_{l}}{\rho} \alpha_{v}\left(1-\alpha_{v}\right) \frac{3}{R_{B}} \sqrt{\frac{2}{3} \frac{\max \left(\left(p_{v}-p\right), 0\right)}{\rho_{l}}} & \text { For } P_{\mathrm{v}} \geq P \\
\dot{m}^{-}=\frac{\rho_{v} \rho_{l}}{\rho} \alpha_{v}\left(1-\alpha_{v}\right) \frac{3}{R_{B}} \sqrt{\frac{2}{3} \frac{\max \left(\left(p-p_{v}\right), 0\right)}{\rho_{l}}} & \text { For } P_{\mathrm{v}} \leq P
\end{array}
$$

The bubbles radius is related to the vapor volume fractions, $\alpha_{\mathrm{v}}$ and bubble number density, $N_{b}$ as:

$$
R_{B}=\left(\frac{\alpha_{v}}{1-\alpha_{v}} \frac{3}{4 \pi} \frac{1}{N_{b}}\right)^{1 / 3}
$$

Where, $N_{b}$ is the only parameter which needs to be specified. It has been taken as $10^{13}$, accordingto Schnerr and Sauer ${ }^{2}$.

Regarding the mixture momentum equation and providing there is no slip between the two phases. It takes the following form:

$$
\frac{\partial(\rho \vec{v})}{\partial t}+\nabla \cdot(\rho \vec{v} \vec{v})=-\nabla p+\nabla \cdot(\mu \nabla \vec{v})+\rho \vec{F}
$$

Where $(F)$ is the external body force. The mixture quantities are defined in the following: 
For volume-averaged mixture viscosity $(\mu)$ :

$\mu=\sum_{q=1}^{n} \alpha_{q} \mu_{q}$

Regarding the turbulence modeling, Large Eddy Simulation (LES) is considered for analyzing transport and mixing of the different scales. For the LES, the large scales are solved directly from the flow equations, while the small turbulent scales are modeled. Spatial scale separation is done by applying a low-pass filter to the governing equations. This set of filtered equations governs the dynamics of the large scales. Spatial fluctuations, lower than a defined filter cutoff length $(\bar{\Delta})$, are resolved by using a sub-model. In this paper consider the cut-off length ( $\bar{\Delta})$ is the grid size and equal to Taylor-scale. The spatial filtering operation of flow variable being a function of time and space is defined by the convolution integral ${ }^{3}$ :

$\bar{f}\left(x_{i}\right)=\int f\left(x_{i}^{\prime}\right) G\left(x_{i}, x_{i}^{\prime}\right) d x_{i}^{\prime}$

By applying the filter on mixture equations (S4) and (S11), the mass and momentum balance equation become:

$\frac{\partial \rho}{\partial t}+\nabla \rho \bar{v}=0$

$\frac{\partial(\rho \bar{v})}{\partial t}+\bar{v} \cdot \nabla \bar{v}=-\nabla \bar{p}+\rho F_{i}+\mu_{e f f} \nabla^{2} \bar{v}-\nabla \cdot \tau^{s g s}$

The SGS stress $\left(\tau^{s g s}=\rho \overline{v \otimes v}-\rho \bar{v} \otimes \bar{v}\right)$ is a new term appearing in LES equations. It accounts for resolved and sub-grid scales interactions. The main task of SGS stress tensor model is to dissipate the energy transferred from turbulent large scales. It can be modeled as follows ${ }^{3}$ :

$\tau^{s g s}-\frac{2}{3} k_{s g s} \nabla \delta=-2 C_{k} k_{s g s}^{1 / 2} \bar{\Delta}(\nabla \bar{S})$

Where $\left(k_{s g s}\right)$ is the subgrid-scale kinetic energy. It can be determined by solving the kinetic energy transport equation.

$\frac{\partial \bar{k}_{s g s}}{\partial t}+\frac{\partial \bar{u}_{j} \bar{k}_{s g s}}{\partial x_{j}}=-\tau_{i j}^{s g s} \frac{\partial \bar{u}_{i}}{\partial x_{j}}-\varepsilon_{s g s}+\frac{\partial}{\partial x_{j}}\left(\frac{\mu_{t}}{\sigma_{k}} \frac{\partial k_{s g s}}{\partial x_{j}}\right)$

Where, 


$$
\mu_{t}=C_{k} k_{s g s}^{1 / 2} \bar{\Delta}
$$

$$
\begin{aligned}
& \varepsilon_{s g s}=C_{\varepsilon} \frac{k_{s g s}^{3 / 2}}{\bar{\Delta}} \\
& \sigma_{k}=1
\end{aligned}
$$

$$
\text { And } \bar{S}_{i j}=\frac{1}{2}\left(\frac{\partial \bar{u}_{i}}{\partial x_{j}}+\frac{\partial \bar{u}_{j}}{\partial x_{i}}\right)
$$

$C_{k}$ and $C_{\mathcal{E}}$ are dynamic constants which are defined in appendix-B.

\section{The Spray Model}

The spray development is performed using the Discrete Droplet Model (DDM). The injected liquid droplets are represented by parcels. These representative parcels move through the gaseous continuous phase. Droplets trajectories are calculated by a Lagrangian formulation that includes hydrodynamic drag, inertia and gravity forces. This is also associated with droplet collision and breakup models. As the problem is isothermal droplets evaporation has not been included.

The governing equations for this approach represented in a vector form, can be summarized as follows:

\section{- Gas Phase:}

Continuity Equation:

$$
\frac{\partial \rho_{g}}{\partial t}+\nabla \cdot\left(\rho_{g} u_{g, i}\right)=0
$$

Momentum Equation:

$$
\frac{\partial\left(\rho_{g} u_{g, i}\right)}{\partial t}+\frac{\partial}{\partial x_{j}}\left(\rho_{g} u_{g, i} u_{g, j}\right)=-\frac{P}{\partial x_{i}}+\mu_{g} \frac{\partial}{\partial x_{i}}\left(\frac{\partial u_{g, i}}{\partial x_{j}}+\frac{\partial u_{g, j}}{\partial x_{i}}\right)+S_{m o m}
$$

Where $\left(S_{\text {mom }}\right)$ is a source term, which accounts for the momentum exchange due to the presence of the droplets. It can be calculated according to equation (S24).

$$
S_{\text {mom }}=m_{d} \cdot\left[\left(\vec{u}_{d, \text { in }}-\vec{u}_{d, \text { out }}\right)+g \cdot\left(1-\frac{\rho_{g}}{\rho_{d}}\right) \cdot\left(t_{\text {in }}-t_{\text {out }}\right)\right]
$$


Where, $\left(m_{d}\right)$ is the mass flow rate of a droplet and $t$ is the time. The quantities with subscripts (in) and (out) refer to the time instants that a droplet enters or leaves a computational control volume.

\section{- Dispersed Phase:}

Droplet momentum equation

$$
\frac{d u_{d}}{d t}=F_{D}\left(u-u_{d}\right)+\frac{g_{x}\left(\rho_{d}-\rho\right)}{\rho_{d}}+F_{x}
$$

Where, $F_{D}\left(u-u_{d}\right)$ is the drag force per unit droplet mass. While the drage force is expressed as follows:

$$
F_{D}=\frac{18 \mu}{\rho_{p} d_{d}^{2}} \frac{C_{D} \operatorname{Re}_{r}}{24}
$$

Where;

$$
\operatorname{Re}_{r}=\frac{\rho d_{d}\left|u_{d}-u\right|}{\mu}
$$

Where, $(\mu)$ is the molecular viscosity of the continuous phase, $\left(d_{d}\right)$ is the droplet diameter, and $\left(\mathrm{Re}_{\mathrm{r}}\right)$ is the relative Reynolds number. The drag coefficient can be taken as:

$$
C_{D}=a_{1}+\frac{a_{2}}{\operatorname{Re}_{r}}+\frac{a_{3}}{\operatorname{Re}_{r}^{2}}
$$

Where $a_{1}, a_{2}, a_{3}$ are constants that apply to smooth spherical droplets over several ranges of $\mathrm{Re}_{\mathrm{r}}$ given by Morsi and Alexander, as illustrated in equations (S29) and (S30).

$$
\begin{array}{ll}
C_{D}=\frac{148.62}{\operatorname{Re}_{r}}-\frac{4.75 \times 10^{4}}{\operatorname{Re}_{r}{ }^{2}}+0.357 & \text { for } 1000.0<\mathrm{Re}_{r}<5000.0, \\
C_{D}=\frac{-490.546}{\operatorname{Re}_{r}}+\frac{57.87 \times 10^{4}}{\operatorname{Re}_{r}{ }^{2}}+0.46 & \text { for } 5000.0<\operatorname{Re}_{r}<10000.0,
\end{array}
$$

Other forces represented in equation (S25) is $\left(F_{x}\right)$ that defines the force required to accelerate the fluid surrounding the droplet (force/unit particle mass), can be take the following form:

$$
F_{x}=\frac{1}{2} \frac{\rho}{\rho_{d}} \frac{d}{d t}\left(u-u_{d}\right)
$$


To predict the dispersion of droplet due to turbulence, the trajectories are solved as stochastic tracking model and the fluid velocity in equation (S25) is considered as mean velocity. The random effect of turbulence on droplet dispersion is considered using the discrete random walk model ${ }^{1}$.

Other models for droplet breakup and collision are also included. This include TAB model for droplet breakup ${ }^{1}$ as well as droplets collision model as developed by O'Rorke ${ }^{1}$. Both models are included in FLUENT software.

\section{Coupling Equations}

To have an accurate solution for the problem, a novel coupling between the in and out flows of the nozzle is needed. As aforementioned the issuing flow is transformed into a spray, which follows a certain droplet size distribution. The distribution has been assumed to follow Rosin-Rammler distribution. The status of the issuing flow and the effective area of the injection nozzle, are considered key parameters in defining the droplet size spectrum, spatial distribution and velocity. This is very much affected by the intensity of cavitation and the following close to the nozzle exit the nozzle, which is transformed into an increase in the turbulent kinetic energy of the issuing flow.

The droplet size distribution is defined by fitting the droplet size to the Rosin-Rammler equation as follow:

$m_{f, d}=e^{-\left(\frac{d_{d}}{\bar{d}_{d}}\right)^{n_{d}}}$

Where $\left(m_{f, d}\right)$ is the mass fraction of droplets with diameter greater than the droplet diameter $\left(d_{d}\right)$, and $\left(\bar{d}_{d}\right)$ is the mean diameter of droplets. Also, $\left(n_{d}\right)$ is the spread parameter which is defined as ${ }^{1}$ :

$n_{d}=\frac{\ln (-\ln m f, d)}{\ln \left(\frac{d_{d}}{\bar{d}_{d}}\right)}$

To define the effect of the high turbulent kinetic energy on the breaking mechanism of the liquid into droplets, an analogy with acoustic wave of ultrasonic atomizers is considered. This was used to find out the initial mean droplet size in equations (S32) and (S33). It can be defined based on the surface tension, density, turbulent frequency and the sub-grid turbulent kinetic energy, as shown in the following equation. 
$\bar{d}_{d}=C_{T}\left(\frac{8 \pi S}{\rho \omega^{2}}\right)^{1 / 3}$

Where, $\omega=\frac{\rho k_{s g s}}{\mu_{t}}$ is the eddy frequency, calculated from sub-grid turbulent kinetic energy, and $\left(C_{T}\right)$ is the proportionality constant, which depends on the fluctuation intensity. It can take the value of 3 according to Kurosawa et.al. ${ }^{4}$. The initial maximum droplet size $\left(d_{d, \max }\right)$ is taken equal to minimum value of either the large eddies scale or nozzle diameter as expressed by equation (S35). On the other hand, the initial minimum droplet size $\left(d_{d, m i n}\right)$ is taken equal to Taylor micro-scale as shown in equation (S36).

$d_{d, \max }=\min \left\{\left(C_{\mu}^{3 / 4} \frac{k_{s g s}^{3 / 2}}{\varepsilon_{s g s}}\right), D_{N}\right\}$

Where, $C_{\mu}=0.09$, and $\left(D_{N}\right)$ is the nozzle diameter.

$d_{d, \min }=\left(\frac{10 v k_{s g s}}{\varepsilon_{s g s}}\right)^{1 / 2}$

\section{Appendix-B}

$C_{k}$ and $C_{\varepsilon}$ are coefficient which determined dynamically by the test-scale. This scale is constructed from the grid-scale by applying a test filter which is characterized by $\left[(\Delta)_{f}=2 \bar{\Delta}\right]$, where the subscript symbol $(f)$ is employed for the test filtering. $C_{k}$ and $C_{\varepsilon}$ are defined as following ${ }^{3}$.

$C_{k}=\frac{1}{2} \frac{L_{i j} \sigma_{i j}}{\sigma_{i j} \sigma_{i j}}$,

$L_{i j}$ is Leonard stress tensor is defined as:

$L_{i j}=\left(\overline{u_{i}} \overline{u_{j}}\right)_{f}-\left(\overline{u_{i}}\right)_{f}\left(\overline{u_{j}}\right)_{f}$

And $\sigma_{i j}=-(\Delta)_{f} k_{t e s t}^{1 / 2}\left(\bar{S}_{i j}\right)_{f}$ 
On the other hand: $C_{\varepsilon}=\frac{\left[\left\langle\left(\frac{\partial \bar{u}_{i}}{\partial x_{j}}\right)\left(\frac{\partial u_{i}}{\partial x_{j}}\right)\right\rangle_{f}-\left\langle\frac{\partial\left(\bar{u}_{i}\right)_{f}}{\partial x_{j}}\right\rangle\left\langle\frac{\partial\left(\bar{u}_{i}\right)_{f}}{\partial x_{j}}\right\rangle\right]}{\left.\left(\mu+\mu_{t}\right)(\Delta)_{f}\right]^{-1} k_{\text {test }}^{3 / 2}}$

Where: $k_{\text {test }}=\frac{1}{2}\left[\left(\bar{u}_{k} \bar{u}_{k}\right)_{f}-\left(\bar{u}_{k}\right)_{f}\left(\bar{u}_{k}\right)_{f}\right]$

\section{References}

(1) Fluent Inc. Fluent User's Guide; 2009.

(2) Schnerr, G. H.; Sauer, J. Physical and Numerical Modeling of Unsteady Cavitation Dynamics. In Fourth international conference on multiphase flow; ICMF New Orleans, 2001; Vol. 1.

(3) Kim, W.-W.; Menon, S.; Kim, W.-W.; Menon, S. Application of the Localized Dynamic Subgrid-Scale Model to Turbulent Wall-Bounded Flows. In 35th aerospace sciences meeting and exhibit; 1997; p 210.

(4) Kurosawa, M.; Futami, A.; Higuchi, T. Characteristics of Liquids Atomization Using Surface Acoustic Wave. In Proceedings of International Solid State Sensors and Actuators Conference (Transducers' 97); IEEE, 1997; Vol. 2, pp 801-804. 